\title{
Substitution-induced phase transition and enhanced multiferroic properties of $\mathrm{Bi}_{1-x} \mathrm{La}_{x} \mathrm{FeO}_{3}$ ceramics
}

\author{
Shan-Tao Zhang, ${ }^{\text {a) }}$ Yi Zhang, Ming-Hui Lu, Chao-Ling Du, Yan-Feng Chen, Zhi-Guo Liu, \\ Yong-Yuan Zhu, and Nai-Ben Ming \\ National Laboratory of Solid State Microstructures and Department of Materials Science and \\ Engineering, Nanjing University, Nanjing 210093, People's Republic of China \\ X. Q. Pan \\ Department of Materials Science and Engineering, University of Michigan, Ann Arbor, \\ Michigan 48109-2136
}

(Received 12 December 2005; accepted 2 March 2006; published online 17 April 2006)

\begin{abstract}
Single-phase, insulating $\mathrm{Bi}_{1-x} \mathrm{La}_{x} \mathrm{FeO}_{3}(\mathrm{BLFO} x, x=0.05,0.10,0.15,0.20,0.30$, and 0.40) ceramics were prepared. An obvious phase transition from rhombohedral to orthorhombic phase was observed near $x=0.30$. It is found that the phase transition destructs the spin cycloid of $\mathrm{BiFeO}_{3}(\mathrm{BFO})$, and therefore, releases the locked magnetization and enhances magnetoelectric interaction. As a result, improved multiferroic properties of the BLFO0.30 ceramics with remnant polarization and magnetization $\left(2 P_{r}\right.$ and $\left.2 M_{r}\right)$ of $22.4 \mu \mathrm{C} / \mathrm{cm}^{2}$ and $0.041 \mathrm{emu} / \mathrm{g}$, respectively, were established.

(C) 2006 American Institute of Physics. [DOI: 10.1063/1.2195927]
\end{abstract}

Multiferroic $\mathrm{BiFeO}_{3}(\mathrm{BFO})$ with simultaneous ferroelectric and $G$-type antiferromagnetic nature are time-honored research subject due to its potential application in devices and the fascinating basic physics of electric and magnetic coupling. ${ }^{1-10}$ In spite of the great achievements on BFO thin films, there are still some problems in bulk BFO, mainly low resistivity and inhomogeneous spin structure. It is accepted that the former problem makes it difficult to draw out the intrinsic ferroelectricity of BFO. ${ }^{6-8}$ The later one leads to the cancellation of macroscopic magnetization (actually, it shows a very weak ferromagnetism according to density functional calculation ${ }^{11}$ ), which prohibits the linear magnetoelectric effect from being observed. ${ }^{12-17}$ Low resistivity is mainly resulted from the valence fluctuation of Fe ions and the existence of second phases, ${ }^{6-8}$ which can be suppressed by optimizing fabricating process or forming BFO-based solid solution. ${ }^{6,8,18-21}$ The inhomogeneous spin structure is resulted from the spatially modulated, cycloidal spin structure with the periodicity of $\sim 620 \AA$, which is incommensurate with the crystallographic lattice parameters. This incommensurate spiral spin structure can be suppressed by strain, ${ }^{1,4}$ high magnetic field ${ }^{13}$ temperature, ${ }^{17}$ and forming BFObased solid solution. ${ }^{18-21}$

The reported solid solution is mainly binary $\mathrm{BFO}-A^{2+} B^{4+} \mathrm{O}_{3}$ and ternary $\mathrm{BFO}-\mathrm{ReFeO}{ }_{3}-A^{2+} B^{4+} \mathrm{O}_{3}$ systems $(\mathrm{Re}=\mathrm{La}$, Pr, and $\mathrm{Dy}, A=\mathrm{Pb}$ and $\mathrm{Ba}$, and $B=\mathrm{Ti})$. However, in these solid solutions, insulating perovskite $A^{2+} B^{4+} \mathrm{O}_{3}$ with stable ferroelectric state is necessary, suggesting that the observed enhancement of ferroelectric property might not be attributed to the magnetoelectric (ME) interaction completely. Therefore, it is difficult to extrapolate and investigate the intrinsic multiferroic properties of BFO. An alternative way is to study new BFO-based solid solution with no other ferroelectric end member except for BFO. Therefore, it might be interesting to prepare and investigate BFO- $\mathrm{LaFeO}_{3}$ (LFO) solid solution. Orthorhombic LFO has homogeneous antiferromagnetic $\left(T_{N}=740{ }^{\circ} \mathrm{C}\right)$ order, ${ }^{22}$

\footnotetext{
${ }^{a)}$ Electronic mail: stzhang@mail.nju.edu.cn
}

which is different from the inhomogeneous one of BFO, and no ferroelectric order. Very recently, epitaxial thin films of BFO-LFO solid solution, or La-substituted BFO $\left(\mathrm{Bi}_{1-x} \mathrm{La}_{x} \mathrm{FeO}_{3}\right.$, BLFO $\left.x, 0 \leqslant x \leqslant 0.15\right)$, have been fabricated on $\mathrm{SrRuO}_{3}$-buffered $\mathrm{SrTiO}_{3}$ substrates by pulsed laser deposition with enhanced ferroelectric and magnetic properties. ${ }^{23}$ However, up to now, no reports on ferroelectric properties and ME interaction of bulk BLFOx system were obtainable in spite of scattered reports on its structures and magnetic properties. $^{16,24}$

In this letter, we report the structures and properties of BLFO $x(0.05 \leqslant x \leqslant 0.40)$ ceramics prepared by conventional solid state reaction followed immediately by quenching processing. A substitution-induced structural phase transition from rhombohedral to orthorhombic is observed near $x$ $=0.30$. The effects of phase transition on multiferroic properties are discussed. The sample preparation will be shown elsewhere.

The structures of the ceramics were characterized by $\mathrm{x}$-ray diffraction (XRD) (Rigaku diffractometer with nickel filtered $\mathrm{Cu} K \alpha$ radiation) and Raman spectroscopy using a Jobin-Yvon LabRam Infinity micro-Raman system with $488 \mathrm{~nm}$ light from Ar ion laser in backscattering geometry. Ferroelectric measurements were performed using RT6000HVS test system (Radiant Technologies) in silicon oil at room temperature while magnetic measurements were carried out using a superconducting quantum interference device (SQUID) (MPMS XL-7) magnetometer.

Figure 1(a) show the XRD patterns of the BLFOx ceramics. It can be seen that the ceramics show single-phase characteristics. Further detailed analysis reveals that some diffraction peaks, e.g., that of (006) diffraction peak shown in Fig. 1(b), become to be weak with increasing $x$ and tend to disappear near $x=0.30$. Note please that the XRD pattern of BLFO0.30 and BLFO0.40 are close to that of orthorhombic $\mathrm{LaFeO}_{3},{ }^{25}$ clearly suggesting a structural phase transition near $x=0.30$. This structure phase transition is consistent with the other report on bulk BLFO $x$ ceramics,${ }^{16,24}$ but different from that in epitaxial BLFO $x$ films. ${ }^{23}$ The reason is 


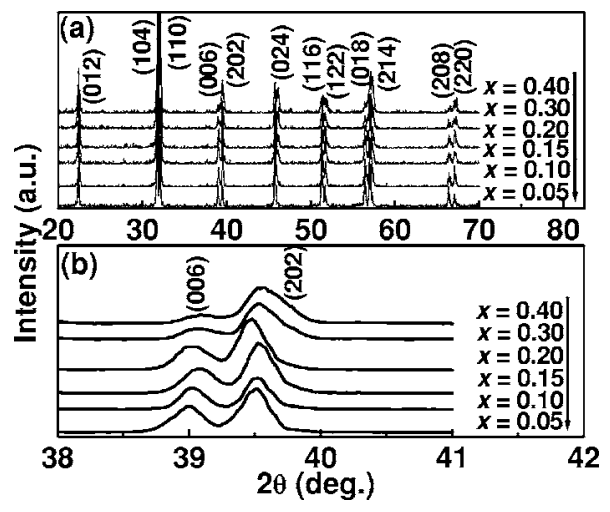

FIG. 1. (a) XRD patterns of the BLFOx ceramics. (b) Subtle XRD patterns of (006) and (202) peaks. The patterns indicate a structural phase transition near $x=0.30$.

that in the thin film form, not only La substitution but also in-plane stress have effects on its structure. $1,3,23$

Figure 2 plots the Raman spectras of BLFO $x$ ceramics. With increasing $x$ from 0 to 0.20 , some vibration modes of BFO show redshift. With further increasing $x$, these modes will either disappear $\left(69 \mathrm{~cm}^{-1}\right)$ or show abnormal blueshift (131 and $168 \mathrm{~cm}^{-1}$ ) accompanied with significantly decreased intensity. It is noted that the two modes at 141 and $172 \mathrm{~cm}^{-1}$ in the BLFO0.30 and BLFO0.40 can also be observed in orthorhombic LFO. ${ }^{25}$ Accordingly, the Raman studies also revealed substitution-induced structural phase transition from rhombohedral to orthorhombic occurs near $x=0.30$.

The spin cycloid of BFO is correlated to its $R 3 c$ structure. Therefore, it is deduced that the structural phase transition might destruct the inhomogeneous spin structure. Room temperature magnetization-magnetic field $(M-H)$ curves of these ceramics were measured with magnetic field of $20 \mathrm{kOe}$ and all ceramics show weak hysteresis loop. The typical ones of the BLFO0.20 and BLFO0.30 are shown in Figs. 3(a) and 3(b), respectively. The measured two time remnant magnetization $\left(2 M_{r}\right)$ values are 0.0020, 0.0041, 0.0056, 0.0084, 0.0410 , and $0.0800 \mathrm{emu} / \mathrm{g}$ for $x=0.05,0.10,0.15,0.20$, 0.30 , and 0.40 , respectively. The $2 M_{r}$ values as the function of $x$ is plotted in Fig. 3(c). Generally, the $2 M_{r}$ value increases with increasing $x$, whereas it is found that the $2 M_{r}$ values of BLFO $x$ with $x \leqslant 0.20$ are significantly less than that of BLFO0.30 and BLFO0.40. These results indicate that with $x \leqslant 0.20$, the La substitution can only suppress but cannot destruct the spin cycloid, leading to limited increase of $2 M_{r}$ value. However, when $x \geqslant 0.30$, the La substitution results in

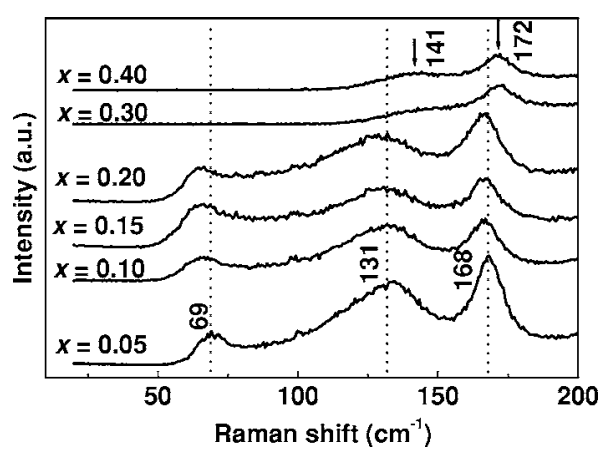

FIG. 2. Raman spectra of the BLFOx ceramics, clearly revealing the phase transition near $x=0.30$.
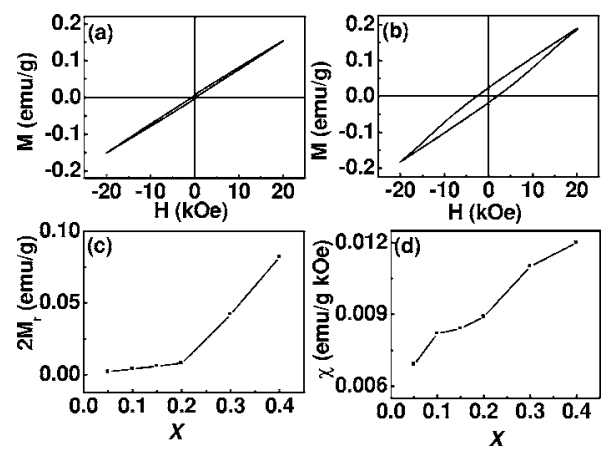

FIG. 3. (a) and (b) show the typical magnetic hysteresis loops of (a) BLFO0.20, and (b) BLFO0.30, respectively. (c) and (d) represent the La composition dependent remnant magnetization and magnetic susceptibility, respectively. The significantly increased magnetization and magnetic susceptibility are attributed to the phase transition-induced destruction of spin cycloid.

structural phase transition wherein the spin cycloid might be destructed and homogeneous spin structure formed, ${ }^{16}$ so that the latent magnetization locked within the cycloid might be released and significant increased $2 M_{r}$ value is observed. Therefore, enhanced ME interaction in BLFO $x$ with $x$ $\geqslant 0.20$ is expected, similar with other $\mathrm{BFO}-\mathrm{PbTiO}_{3}$-based solid solution. ${ }^{21}$ It should be noted that the further increased $2 M_{r}$ and magnetic susceptibility of BLFO0.40 compared to that of BLFO0.30 do not mean that the spin cycloidal structure is not fully destructed in BLFO0.30. Some charged point defects induced by quenching processing in orthorhombic BLFO0.40, as will be discussed below, might be responsible for the observed increasing.

According to Landau-Ginzburg theory, ${ }^{13,21}$ destruction of spin cycloid of BFO leads to enhanced ME interaction, which can improve both the effective magnetic susceptibility and ferroelectric remnant polarization of multiferroics. The effective magnetic susceptibility $(\chi)$ can be determined from the slop of the $M-H$ curve at $H=0 .{ }^{21}$ The $x-\chi$ curve of the BLFO $x$ ceramics is plotted in Fig. 3(d). Susceptibility increases with increasing $x$. However, the abruptly increase near $x=0.30$ should be attributed directly to the enhanced $\mathrm{ME}$ interaction by the destruction of spin cycloid.

Accordingly, it is expected that BLFO0.30 should have improved ferroelectric property. The typical hysteresis loops of BLFO0.20, BLFO0.30, and BLFO0.40 are shown in Figs. 4(a)-4(c), respectively. The loops of BLFO0.05, BLFO0.10, and BLFO0.15 are well saturated (not shown), similar with that of BLFO0.20 and BLFO0.30. Here, it should be men-

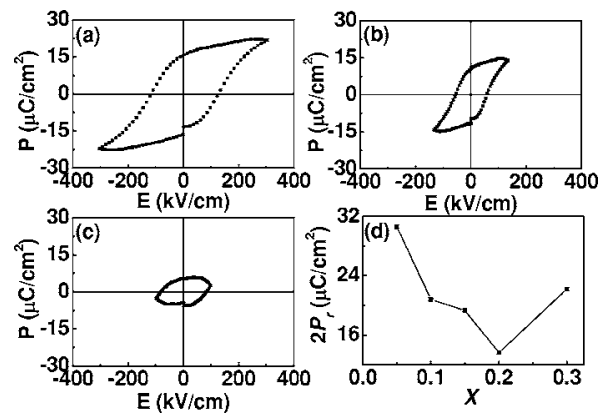

FIG. 4. Typical ferroelectric hysteresis loops of (a) BLFO0.20, (b) BLFO0.30, and (c) BLFO0.40. (d) plots the La composition dependent remnant polarization. The enhanced $\mathrm{ME}$ interaction due to the destruction of cycloidal spin is responsible for the improved ferroelectricity of BLFO0.30. 
tioned that these values are obtained with different electric fields of 200, 230, 255, and $305 \mathrm{kV} / \mathrm{cm}$, respectively. For the BLFO0.40 ceramics, the low resistivity makes the ferroelectric measurement difficult. Actually, as shown in Fig. 4(d), if measured with comparable electric field, the obtained $2 \mathrm{P}_{r}$ will decrease with increasing $x$ from 0.05 to 0.20 and then tend to increase with $x=0.30$, which corresponds to the above described structural phase transition. Please note in Fig. 4(d), the data of BLFO $x$ with $x \leqslant 0.20$ are obtained with $200 \mathrm{kV} / \mathrm{cm}$ electric field whereas that of BLFO0.30 is obtained with $135 \mathrm{kV} / \mathrm{cm}$ electric field. However, even in this case, the $2 \mathrm{P}_{r}$ of BLFO0.30 is 1.7 times larger than that of BLFO0.20, indicating the greatly improved ferroelectricity of BLFO0.30.

By considering Figs. 3 and 4 simultaneously, it is found that for BLFO $x$, if the spin cycloid of BFO cannot be destructed, the ferroelectricity tends to decrease with increasing $x$, however, if it is destructed, the ferroelectricity tends to increase. Therefore, the improved ferroelectricity of BLFO0.30 should be attributable to the enhanced ME interaction due to the destruction of cycloidal spin structure. Though on the other hand, phase transition can also cause the change of polarization, it might not be a sound possibility responsible for the above observation because it has been revealed in (111) and (001)-epitaxial BFO thin films, the phase transition between rhombohedral and nomoclinical structure, which is close to orthorhombic structure $\left(\beta=89.2^{\circ}\right)$, has no significant effect on polarization. ${ }^{3,5}$

Another feature about the hysteresis loops should be mentioned is that for each ceramics, further increasing electric field will lead to electric breakdown. That is, with increasing $x$ from 0 to 0.40 , the breakdown electric field increases, reaching the maximum at $x=0.20$, and then decreases, this means the resistivity of the ceramics increases when $x \leqslant 0.20$ and then decreases. According to the phase diagram of $\mathrm{Bi}_{2} \mathrm{O}_{3}-\mathrm{Fe}_{2} \mathrm{O}_{3}$ system and related reports, ${ }^{26}$ the heated rhombohedral BFO can only be a stable phase in a temperature window. If the heated rhombohedral BFLO $x-(x$ $\leqslant 0.20$ ) in this window is cooled naturally, there should also be local deviation from stoichiometry and the formation of $\mathrm{Fe}^{2+}$, both of which play a detrimental role in determining resistivity of BFO-based ceramics. Quenching processing may suppress the formation of second phases and $\mathrm{Fe}^{2+}$ by freezing and keeping the high temperature stoichiometric BLFO $x$ form. However, as for orthorhombic BLFO $x(x$ $\geqslant 0.30$ ) there is no such a naturally formed impurity phases and $\mathrm{Fe}^{2+}$ during cooling, so, quenching may have negative instead of positive effect on resistivity of the ceramics by preserving the point defect formed at high temperature. The effect of quenching on structure and phase purity of BFObased ceramics is discussed separately.

In summary, single-phase BLFO $x$ ceramics have been prepared and their structures and properties were studied. A phase transition from rhombohedral to orthorhombic is observed near $x=0.30$. This structural phase transition destructs the spin cycloid, releases a locked magnetization, and en- hances the ME interaction, which results in significantly improved ferroelectric and magnetic properties of BFLO0.30 with $2 \mathrm{P}_{r}$ of $22.4 \mu \mathrm{C} / \mathrm{cm}^{2}$ and $2 M_{r}$ of $0.041 \mathrm{emu} / \mathrm{g}$.

The authors acknowledge the finical support from the National Nature Science Foundation of China (Contract Nos. 110404012, 50225204, 10374048 and 50428202) and the State Key Program for Basic Research of China (2001CB610404) and the program for Changjiang Scholars and Innovative Research Team in University (PCSIRT).

${ }^{1}$ J. Wang, J. B. Neaton, H. Zheng, V. Nagarajan, S. B. Ogale, B. Liu, D. Viehland, V. Vaithyanathan, D. G. Schlom, U. V. Waghmare, N. A. Spaldin, K. M. Rabe, M. Wuttig, and R. Ramesh, Science 299, 1719 (2003).

${ }^{2}$ K. Y. Yun, M. Noda, M. Okuyama, H. Saeki, H. Tabata, and K. Saito, J. Appl. Phys. 96, 3399 (2004).

${ }^{3}$ J. Li, J. Wang, M. Wuttig, R. Ramesh, N. G. Wang, B. Ruette, A. P. Pyatakov, A. K. Zvezdin, and D. Viehland, Appl. Phys. Lett. 84, 5261 (2004).

${ }^{4}$ F. Bai, J. Wang, M. Wuttig, J. Li, N. Wang, A. P. Pyatakov, A. K. Zvezdin, L. E. Cross, and D. Viehland, Appl. Phys. Lett. 86, 032511 (2005).

${ }^{5}$ G. Xu, H. Hiraka, G. Shirane, J. Li, J. Wang, and D. Viehland, Appl. Phys. Lett. 86, 182905 (2005).

${ }^{6}$ M. M. Kumar, V. R. Palkar, K. Srinivas, and S. V. Suryanarayana, Appl. Phys. Lett. 76, 2764 (2000).

${ }^{7}$ Y. P. Wang, L. Zhou, M. F. Zhang, X. Y. Chen, J. M. Liu, and Z. G. Liu, Appl. Phys. Lett. 84, 1731 (2004).

${ }^{8}$ A. K. Pradhan, K. Zhang, D. Hunter, J. B. Dadson, G. B. Loutts, P. Bhattacharya, R. Katiyar, J. Zhang, and D. J. Sellmyer, J. Appl. Phys. 97, 093903 (2005)

${ }^{9}$ S. Iakovlev, C.-H. Solterbeck, M. Kuhnke, and M. Es-Souni, J. Appl. Phys. 97, 094901 (2005).

${ }^{10}$ W. Eerenstein, F. D. Morrison, J. Dho, M. G. Blamire, J. F. Scott, and N. D. Mathur, Science 307, 1203a (2005).

${ }^{11}$ C. Ederer and N. A. Spaldin, Phys. Rev. B 71, 060401(R) (2005).

${ }^{12}$ I. Sosnowska, T. Peterlin-Neumaier, and E. Steichele, J. Phys. C 15, 4835 (1982).

${ }^{13}$ B. Ruette, S. Zvyagin, A. P. Pyatakov, A. A. Bush, J. F. Li, V. I. Belotelov, A. K. Zvezdin, and D. Viehland, Phys. Rev. B 69, 064114 (2004).

${ }^{14}$ Yu. F. Popov, A. M. Kadomtseva, S. S. Drotov, D. V. Belov, G. P. Worob'ev, P. N. Makhov, and A. K. Zvezdin, Low Temp. Phys. 27, 478 (2001).

${ }^{15}$ I. Sosnowska, W. Schäfer, W. Kockelmann, K. H. Andersen, and I. O. Troyanchuk, Appl. Phys. A: Mater. Sci. Process. 74, S1040 (2002).

${ }^{16}$ A. V. Zalesskii, A. A. Frolov, T. A. Khimich, and A. A. Bush, Phys. Solid State 45, 141 (2003).

${ }^{17}$ A. V. Zalesskii, A. K. Zvezdin, A. A. Frolov, and A. A Bush, JETP Lett. 71, 465 (2000).

${ }^{18}$ J. S. Kim, C. I. Cheon, C. H. Lee, and P. W. Jang, J. Appl. Phys. 96, 468 (2004).

${ }^{19}$ J. S. Kim, C. I. Cheon, Y. N. Choi, and P. W. Jang, J. Appl. Phys. 93, 9263 (2003).

${ }^{20}$ J. R. Cheng, N. Li, and L. E. Cross, J. Appl. Phys. 94, 5153 (2003).

${ }^{21}$ N. Wang, J. Cheng, A. Pyatakov, A. K. Zvezdin, J. F. Li, L. E. Cross, and D. Viehland, Phys. Rev. B 72, 104434 (2005).

${ }^{22}$ J. Lüning, F. Nolting, A. Scholl, H. Ohldag, J. W. Seo, J. Fompeyrine, J.-P. Locquet, and J. Stöhr, Phys. Rev. B 67, 214433 (2003).

${ }^{23}$ D. Lee, M. G. Kim, S. Ryu, H. M. Jang, and S. G. Lee, Appl. Phys. Lett. 86, 222903 (2005).

${ }^{24}$ Z. V. Gabbasova, M. D. Kuz'min, A. K. Zvezdin, I. S. Dubenko, V. A. Murashov, D. N. Rakov, and I. B. Krynetsky, Phys. Lett. A 158, 491 (1991).

${ }^{25}$ M. Popa, J. Frantti, and M. Kakihana, Solid State Ionics 154, 135 (2002).

${ }^{26}$ J. Yu, N. Koshikawa, Y. Arai, S. Yoda, and H. Saitou, J. Cryst. Growth 231, 568 (2001). 